\title{
A novel phantom design for brachytherapy quality assurance
}

\author{
S. Gholami ${ }^{1}$, H.R. Mirzaei $^{2}{ }^{*}$, A. Jabbary Arfaee ${ }^{2}$, R. Jaberi ${ }^{3}$, \\ H.A. Nedaie ${ }^{1,3}$, S. Rabi Mahdavi4, E. Rajab Bolookat ${ }^{2}$, A.S. Meigooni5
}

\author{
${ }^{1}$ Department of Medical Physics and Biomedical Engineering, Faculty of Medicine, Tehran University of Medical \\ Sciences, Tehran, Iran \\ ${ }^{2}$ Radiation Oncology Department, Shohada e Tajrish Hospital, Cancer Research Center, Shahid Beheshti University \\ of Medical Sciences, Tehran, Iran \\ ${ }^{3}$ Radiation Oncology Department, Cancer institute, Tehran University of Medical Sciences, Tehran, Iran \\ ${ }^{4}$ Department of Medical Physics, Iran University of Medical Sciences, Tehran, Iran \\ ${ }^{5}$ Comprehensive Cancer Centers of Nevada, Las Vegas, Nevada, United States
}

\section{Technical note}

\section{*Corresponding author: \\ Dr. Hamidreza Mirzaei, \\ E-mail: \\ mirzaei65@yahoo.com}

Revised: Feb. 2015

Accepted: April 2015

Int. J. Radiat. Res., January 2016; 14(1): $67-71$

DOI: 10.18869 /acadpub.ijrr.14.1.67

\begin{abstract}
Background: One major challenge in brachytherapy is to verify the accuracy of dose distributions calculated by the treatment planning system. In this project, a new phantom design has been introduced for quality assurance of dose distributions in gynocological (GYN) brachytherapy implants using EBT GafChromic film. Materials and Methods: This phantom has been designed and fabricated from 90 slabs of $18 \times 16 \times 0.2 \mathrm{~cm}^{3}$ Perspex to accommodate a tandem and ovoids assembly, which is normally used for GYN brachytherapy treatment. In addition, this phantom design is allowing the use EBT GafChromic films for dosimetric verification of GYN implants with Cs-137 Selectron LDR system. With this assembly, GafChromic films were exposed using a plan designed to deliver 2.5 Gy dose to point " $A$ " in Manchester system for tandem and ovoids configurations and to deliver $1.5 \mathrm{~Gy}$ of dose to $0.5 \mathrm{~cm}$ distance from the lateral surface of ovoids for using ovoid-pair. The measured dose distributions with GafChromic films were compared with the TPS isodose lines both numerically and spatially. For a quantitative analysis of the results, the measured doses values at several points of interest were evaluated with the treatment planning data and values obtained following the TG-43 dose calculation formalism. Results: The results of these investigations have indicated that the new phantom design enables us to measure differences of greater than $\pm 6 \%$ for LDR brachytherapy GYN treatments. Conclusion: The new phantom design could be utilized for the QA procedure of the brachytherapy remote after loading systems to confirm the accuracy of dose distribution in GYN implants.
\end{abstract}

Keywords: $Q A$ brachytherapy phantom, film dose measurements, Selectron LDR system, TG-43.

\section{INTRODUCTION}

In brachytherapy, radioactive sources are placed near or within the tumor volume in order to maximize the dose delivery to the tumor and minimize the dose to the surrounding normal tissues. GYN brachytherapy treatments for cervix, vaginal and endometrial cancers have been commonly used for several decades (1). Accuracy of dose calculation by the treatment planning systems plays a vital role on the success of these brachytherapy treatments. As 
brachytherapy techniques become more complex, the quality assurance (QA) techniques have to be modified to provide a sound and practical method of verification of treatment delivery system. The progress is needed for QA of complex brachytherapy treatment techniques, particularly when it involves treatment with complex low dose rate (LDR) or high dose rate (HDR) gynecologic (GYN) systems. The QA tests are required when a new planning system has been introduced for clinical application. For example, recently a new high dose rate brachytherapy system has been introduced based on ${ }^{60}$ Co radionuclide (GZP6 machine) (2). This system has its own planning system which may require QA tests before it is implemented in clinical procedures.

Experimental verification of the dose accuracy is one possible QA procedures for determination of any over-dose or under-dose area in the brachytherapy planning volume. One important criteria for these experimental setups is to have a 2D or 3D dosimeter with high spatial resolution such as GafChromic ${ }^{\mathrm{TM}}$ (also known as Radiochromic ${ }^{\mathrm{TM}}$ : previously distributed by International Speciality Product (ISP) but it is presently distributed by the Ashland Refining Company) films. Different investigators had demonstrated the usefulness of the EBT GafChromic $^{\mathrm{TM}}$ films for brachytherapy source dosimetry $(3,4)$. These films can be cut to the shape of the experimental geometry for the best representation of the dosimetric setup.

In this project, a new phantom has been designed and fabricated for dosimetric verification of the GYN implants with low dose rate (LDR) or high dose rate (HDR) systems, using EBT GafChromic ${ }^{\mathrm{TM}}$ film. The accuracy and functionality of this phantom design has been examined using a well stablished LDR ${ }^{137} \mathrm{Cs}$ Selectron remote afterloading system, which is distributed by Nucletron (Nucletron BV, Veenendaal, The Netherlands) (5).

This phantom design enable us to verify the accuracy of the dose distributions around various combinations of tandem and ovoid applicators. The results of the measured data with this device were compared with calculated values from treatment planning systems and also with hand calculations based on TG-43 formalism.

\section{MATERIALS AND METHODS}

\section{LDR Cs-137 selectron remote after-loading system}

In the present study, the reference points for dose prescription were selected based on Manchester system (6) and ICRU38 recommendation (7). A tandem and ovoids combination was selected, with $5 \mathrm{~cm}$ long on tandem \#1 and large size ovoids (3cm diameter). With this assembly a set of 17 active and dummy pellets were used in tandem and 8 pellets in each ovoid. From the pellets in tandem, numbers $1,4,7,8,9,10,11,12,13$ were chosen to be active. In each ovoid, pellets numbers 2 to 7 were considered active. The remainder pellets were non-active, in order to deliver 2.5 Gy of dose to point "A" in Manchester system (6) in 1.08 hours. For using ovoid- pair geometry, large size $(3 \mathrm{~cm}$ diameter $)$ ovoids were selected. Again, pellet numbers 2 to 7 were considered active and the remainders were non-active to deliver $1.5 \mathrm{~Gy}$ of dose to $0.5 \mathrm{~cm}$ lateral distance from surface of ovoids in 1.01 hours. This dose represents the same dwell positions and dwell times as the ovoid portion of the tandem and ovoids assembly. The Nucletron (PLATO v.11.4) treatment planning system (TPS) was utilized for calculation of the dose distribution by this system ${ }^{(5)}$.

\section{New phantom design and dose measurements technique}

The new phantom has been designed and fabricated from 90 slabs of $18 \times 16 \times 0.2 \mathrm{~cm}^{3}$ Perspex to accommodate GYN applicators (tandem and ovoid). This configuration enables us to verify the dose distributions around the applicator with high spatial resolution. Figure 1 shows the schematic diagram of this phantom from different perspective views and also the picture of the sample applicator assembly placed in the phantom.

The thin layers of the slabs were carefully 
machined to accommodate the GafChromic ${ }^{\mathrm{TM}}$ films in between the slabs for radiation dosimetry. Also, the phantom design could be adjusted with some adaptive inserts to accommodate other ovoid sizes.

The points of interests include points " $\mathrm{A}$ " and "B" in Manchester system for tandem and ovoids combination and points "D" and "F" for ovoid-pair, as shown in figure 2 , which are located at $0.5 \mathrm{~cm}$ and $1.0 \mathrm{~cm}$ distances from the lateral surface of ovoid, respectively. Also, the measured isodose lines were compared with the treatment planning data. Figure 3 shows the picture of the irradiated films that were cut in various sizes and shape to match the curvature and location of the applicator.

\section{Film dosimetry procedure}

For a qualitative evaluation of the new phantom design, several layers of GafChromic ${ }^{\mathrm{TM}}$ film have been irradiated using the tandem and ovoid combination and ovoid-pair geometry. The irradiated films for any implant geometry and calibration procedures were scanned with the same technique after 24 hours from exposure time. As suggested in the guideline of the GafChromic ${ }^{\mathrm{TM}}$ films, the irradiated films for

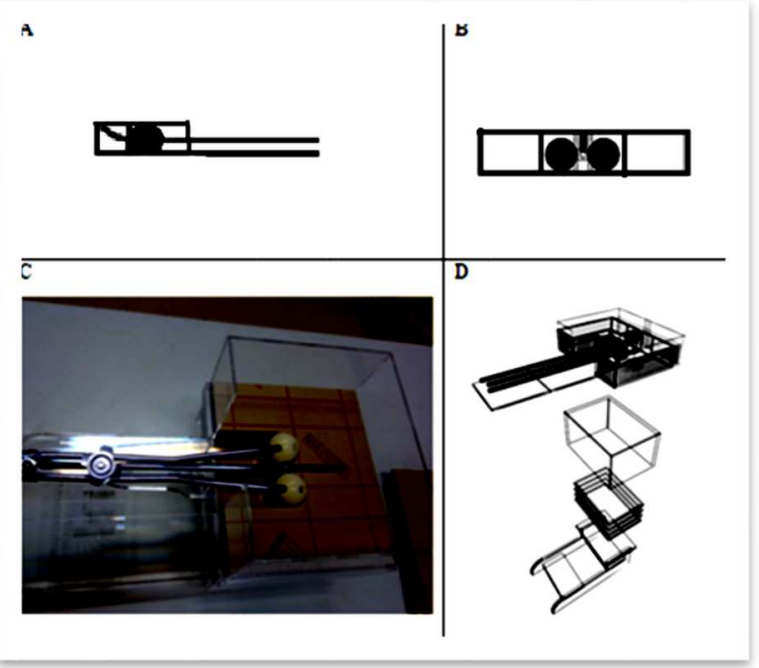

Figure 1. Schematic diagrams of the new phantom design with the lateral view of the GYN applicator (A), Axial View (B), a photograph of the GYN tandem and ovoid applicator placed within the phantom $(C$, and schematics of different layers of the phantom and applicator (D). (A patent for this phantom design has been submitted to the office of Industrial property with registration number of 73752). both calibration and measurement process were scanned in the landscape orientation (8).

Scanned EBT GafChromic ${ }^{\mathrm{TM}}$ films were transformed to IMAGEJ software (Java-based image processing program, National Institute of Health) (9). The dose value of reference point according to its position in the film was calculated. Both the calibrated and experimental film scanning have been performed using 100 dpi resolutions. The pixel value was obtained from IMAGEJ software. The maximum possible reading of the scanner was $\mathrm{I}_{0}=65535$.

Net optical density (NOD) is obtained by subtracting the reading for the base fog (OD of un-irradiated film) form the measured optical density (10).
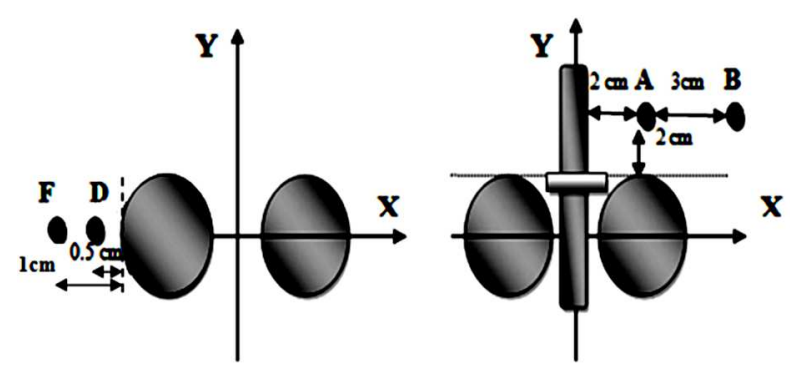

Figure 2. Schematic diagram of the tandem-ovoids and ovoid-pair applicators and calculated points relative to the applicators (Graphs are not to the scale).

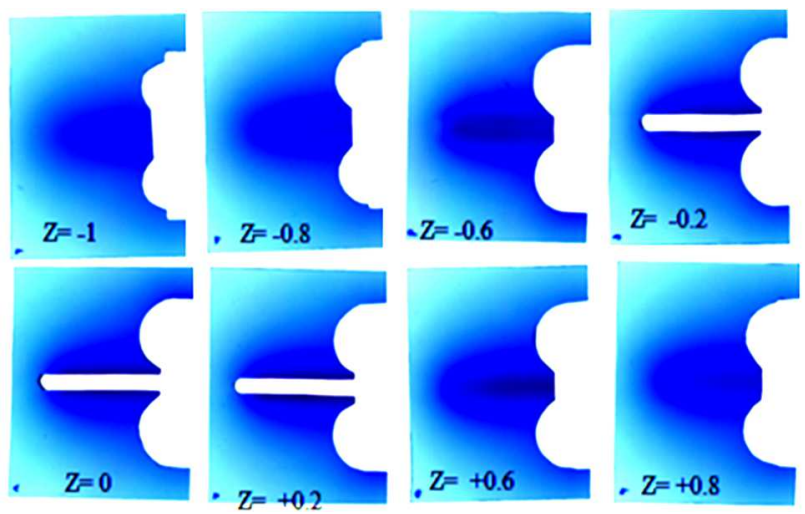

Figure 3. Different slices of EBT-1 films, exposed to the radiation from tandem and ovoid Assembly. These films were cut in different size and shape to match the curvature of the applicators and were placed various locations in the phantom. The labels on the films are used to identify the orientation and position of the films within the phantom. $(\mathrm{Z}=0$ represents a surface that transverse axes of film are on ovoids center).

Int. J. Radiat. Res., Vol. 14 No. 1, January 2016 


\section{TG-43 calculation}

For LDR ${ }^{137}$ Cs Selectron remote after loading unit film dosimetries with the new phantom have been completed at multiple levels relative to the GYN applicators. TG-43 calculations were considered for some interesting points to verify the agreement between calculated dose value from film dose measurements and dose value in treatment planning.

TG-43 formalism was employed depend on dosimetric parameters of ${ }^{137} \mathrm{Cs}$ source. These parameters consist of air kerma strength, dose rate constant, geometry function, radial dose function and anisotropy function. TG-43 parameters of ${ }^{137} \mathrm{Cs}$ Selectron LDR brachytherapy sources were considered from Sina's Monte Carlo simulation (11). Dose rate constant value for this unit was $1.102\left(\mathrm{cGyh}^{-1} \mathrm{U}^{-1}\right)$.

\section{Uncertainty analysis}

The accurate results of GafChoromic ${ }^{\mathrm{TM}}$ film dosimetry significantly depend on the position accuracy of the source with respect to the point of interest (12). A special attention was paid to the distances between the phantom's edge and edge of the applicator and the film.

The distance between the phantom slabs' edge and the applicators is 95 micrometer. Moreover, there is a $0.1 \mathrm{~mm}$ gap between edge of the machined film and the phantom's edge. Also, there is a 0.5 millimeter error in determining the position of points of interest.

With considering the slope of dose profile at level of point "A" from IMAGEJ software, we can estimate the uncertainty of total dose due to the uncertainty of the coordinate of the points of interests.

\section{RESULTS}

\section{EBT GafChromic film calibration}

The calibration curve of the EBT GafChromic $^{\mathrm{TM}}$ film response has $\pm 5 \%$ uncertainty. Moreover, a $2 \%$ of uncertainty is attributed due to the energy difference between 60Co (used for calibration) and 137Cs. The uncertainty of source-to-film positioning is $4.25 \%$. Table 1 shows the uncertainties analysis for dose measurements in this study.

\section{Results of selectron LDR brachytherapy measurements}

The value of reference air kerma rate for TG-43 dose calculation is extracted from treatment planning system which was $87.88 \times 10^{-6} \mathrm{Gym}^{2} \mathrm{~h}^{-1}$. The quantitative analysis of the measured data for Selectron ${ }^{137} \mathrm{Cs}$ LDR source and TG-43 dose calculation is shown in table 2 . These results show a good agreement (about 6\%) between the film dose measurements, TG-43 dose calculation and treatment planning using designed phantom.

Table 1. Total uncertainty analysis for film dose measurements.

\begin{tabular}{|c|c|}
\hline Source of uncertainty & $\begin{array}{c}\text { Percent of } \\
\text { uncertainty }\end{array}$ \\
\hline Source-to-film positioning $(\Delta \mathrm{a})$ & $4.25 \%$ \\
\hline Calibration curve fit $(\Delta \mathrm{b})$ & $5 \%$ \\
\hline $\begin{array}{c}\text { Difference between film calibration source } \\
\text { And Brachytherapy source }(\Delta \mathrm{c})\end{array}$ & $2 \%$ \\
\hline Total uncertainty $\left(\sqrt[2]{\Delta a^{2}+\Delta b^{2}+\Delta c^{2}}\right)$ & $6.86 \%$ \\
\hline
\end{tabular}

Table 2. Comparison between the measured and calculated dose values at different points around a typical applicators in GYN implant of Selectron ${ }^{137}$ Cs LDR source.

\begin{tabular}{|c|c|c|c|c|c|c|}
\hline Points & $\begin{array}{c}\text { Dose- } \\
\text { treatment } \\
\text { planning } \\
\text { (cGy) }\end{array}$ & $\begin{array}{c}\text { Dose-TG- } \\
\mathbf{4 3} \text { (cGy) }\end{array}$ & $\begin{array}{c}\text { Dose- Film } \\
\text { (cGy) }\end{array}$ & $\begin{array}{c}\text { Difference } \\
\text { between } \\
\text { planning and } \\
\text { TG-43 } \\
\text { calculation (\%) }\end{array}$ & $\begin{array}{c}\text { Difference } \\
\text { betference } \\
\text { between film } \\
\text { dosimetry and } \\
\text { TG-43 } \\
\text { calculation (\%) }\end{array}$ & $\begin{array}{c}\text { betwen film } \\
\text { dosimetry and } \\
\text { treatment } \\
\text { planning (\%) }\end{array}$ \\
\hline Point A & 250.0 & 255.4 & 244.0 & $-2.1 \%$ & $-4.4 \%$ & $-2.4 \%$ \\
\hline Point B & 70.0 & 71.1 & 67.0 & $-1.5 \%$ & $-4.8 \%$ & $-4.3 \%$ \\
\hline Point D & 150.0 & 153.2 & 145.8 & $-2.0 \%$ & $-6.0 \%$ & $-2.8 \%$ \\
\hline Point F & 100.0 & 102.0 & 95.8 & $-1.9 \%$ & & $-4.2 \%$ \\
\hline
\end{tabular}

Int. J. Radiat. Res., Vol. 14 No. 1, January 2016 


\section{DISCUSSION AND CONCLUSION}

A sophisticated device for verification of the accuracy of dose distribution by a 3D treatment delivery system in brachytherapy is greatly desirable. In this project, a novel phantom was designed and fabricated for QA of the dose distributions around GYN brachytherapy treatments, using EBT GafChromic ${ }^{\mathrm{TM}}$ film. The accuracy of this device has been examined with LDR selectron remote afterloading system with ${ }^{137}$ Cs sources. The result of using this phantom for Selectron system shows good agreement between the film-measured dose values, TG-43 based calculated data and treatment planning values (about 6\%). Since the accuracy of the measurements was checked at some reference points and also for isodose lines, it is predicted to obtain good results for other points as well. In table 2, despite the fact that same formalism has been used for the hand calculation with TG-43 and treatment planning calculations, the differences may indicate the uncertainties due to the digitization of positions for the points of interests in the treatment planning system.

\section{ACKNOWLEDGMENT}

The Radiotherapy Department of Shahid Beheshti University sponsored the purchase of the phantom materials and films used in the investigations. The authors would like to present their appreciation for Dr Mehdi Ghorbani for his invaluable contributions in this project. In addition, the editorial suggestions by Dr Courtney Knaup are greatly appreciated.

\section{Conflicts of interest: none to declare.}

\section{REFERENCES}

1. Gerbaulet A, Potter R, Mazeron J, Van Limbergen E, Editors (2002) The GEC ESTRO handbook of brachytherapy.
Brussels: ESTRO [cited 2012 Feb1]. Available from :http:// estro-education.org/publications/Documents/GEC\% 20ESTRO\%20Handbook\%20of\%20Brachytherapy. html

2. Mesbahi A (2008) Radial dose functions of GZP6 intracavitary brachytherapy 60Co sources: treatment planning system versus Monte Carlo calculations. Iran J Radiat Res, 5 (4): 181-6.

3. Niroomand-Rad A, Blackwell CR, Coursey BM, Gall KP, Galvin JM, McLaughlin WL, Meigooni AS, Nath R, Rodgers J E, Soares CG (1998) Radiochromic film dosimetry: Recommendations of AAPM Radiation Therapy Committee Task Group 55. Med Phys, 25: 2093-116.

4. Chiu-Tsao ST, Medich D, Munro J (2008) The use of new GAFCHROMIC EBT film for ${ }^{125}$, seed dosimetry in Solid Water phantom. Med Phys, 35(8): 3787- 99.

5. Fragoso $M$, Love PA, Verhaegen $F$, Nalder C, Bidmead AM, Leach M, Webb S (2004) The dose distribution of low dose rate $\mathrm{Cs}-137$ in intracavitary brachyther-apy: comparison of Monte Carlo simulation, treatment planning calculation and polymer gel measurement. Phys Med Biol, 49(24): 5459-74.

6. Tod M, Meredith WJ (1953) Treatment of cancer of the cervix uteri-a revised Manchester method. Br J Radiol, 26: 252-7.

7. International Commission on Units and Measurement (ICRU) Dose and Volume Specification for Reporting Intracavitary Therapy in Gynecology. ICRU Report No.38.1985; Bethesda (MD): ICRU.

8. ISP (International specialty products). Handling guide of GafChromic films .Available from: http:// online1.ispcorp.com/_layouts/Gafchromic/content/ products/ebt2/pdfs/ GAFCHROMICEBT2ScanHandlingGuide.pdf.

9. ImageJ Documentation Wiki . Available from: http:// imagejdocu.tudor.lu/

10.Chan M, Zhang Q, Li J, Parhar P, Schupak K, Burman CH (2012) The Verification of iPlan commissioning by Radiochromic EBT2 Films. IJMPCERO, 1: 1-7.

11.Sina S, Faghihi R, Meigooni AS, Mehdizadeh S, Mosleh Shirazi MA, Zehtabian M (2011) Impact of the vaginal applicator and dummy pellets on the dosimetry parameters of Cs-137 brachytherapy source. J Appl Clin Med Phys, 12(3): 183-93.

12.Aldelaijan S, Mohammad H, Tomic N, Liang L $H$, Deblois F, Sarfehnia A, Abdel-Rahman W, Seuntjens J, Devic S (2011) Radiochromic film dosimetry of HDR ${ }^{192}$ Ir source radiation fields. Med Phys, 38(11): 6074-83.

13. Lipińska J, Zwierzchowski G (2006) Dosimetric verification of the dose distribution in pulsed dose rate brachytherapy. Rep Pract Oncol Radiother, 11: 223-8.

14. Kohr P and Siebert A (2007) Quality assurance of brachytherapy afterloaders using a multi-slit phantom. Phys Med Biol, 52: N387-N91. 
